\title{
Éditorial
}

\section{«Les rayons de Roentgen sont comme la lance d'Achille, ils blessent et ils guérissent »}

Le titre de cet éditorial, emprunté à Antoine Béclère (1904), est aussi l'incipit du livre de notre ancien président, le professeur Jean-Marc Cosset : Des rayons contre le cancer - Tout savoir sur la radiothérapie (Robert Laffont, 2008, ISBN : 978-2221-11070-6). Il pourrait paraître, pour certains d'entre vous, une provocation dans un journal consacré à la radioprotection. Mais il n'en est rien, rassurez-vous, il me sert simplement à dédier, une fois n'est pas coutume, un éditorial à une revue de livres.

Pour moi, 2008 restera marqué par la publication de cet excellent ouvrage.

Ce livre est remarquable et se lit d'une traite ou presque. Il présente tout d'abord ce fléau que reste «le cancer»- et principalement les cancers du sein et de la prostate - puis nous explique comment la radiothérapie contribue aux progrès thérapeutiques considérables qui nous permettent de moins redouter cette terrible maladie. Le talent pédagogique du professeur est magnifié par le ton de ce livre, très personnel. Jean-Marc n'hésite pas à parler de son expérience vécue, en tant que radiothérapeute renommé d'une part, mais aussi en tant qu'ancien malade puisque, comme il le dit lui-même, « il s'en est allé explorer l'autre côté de la barrière »... Ceci donne à cet ouvrage une dimension humaine loin des habituels traités un peu secs sur ces dramatiques questions.

Jean-Marc nous relate aussi les difficultés rencontrées par la profession, et ce avec une totale honnêteté. Il nous avoue que, si par le passé, il pouvait suppléer au pied levé à l'absence d'un technicien, aujourd'hui les machines sont tellement sophistiquées qu'elles requièrent des manipulateurs aguerris à ces nouvelles techniques.

Loin de la langue de bois, il relate avec humour comment il a rendu publiques les archives de l'Institut Curie portant sur les incidents et accidents. Je me rappelle qu'à l'époque, il n'était guère soutenu et c'est un euphémisme ! Il ne fait jamais bon d'être précurseur! Connaître les incidents nous permet pourtant d'avoir un retour d'expérience des plus utiles. Cette action, il l'a également menée au sein du comité 3 de la CIPR alors présidé par le talentueux Fred Mettler. Et, en conclusion, il n'occulte pas le difficile problème de la communication, nécessaire certes, mais qui ne doit pas conduire à une crainte, voire à un refus, des traitements de radiothérapie. Là, nous avons encore beaucoup à faire.

Bref, je pense que cet ouvrage méritait un éditorial et un coup de chapeau.

DOI: 10.1051/radiopro/2009002

RADIOPROTECTION - VOL. 44 - $\mathrm{N}^{\circ} 1$ (2009) 
ÉDITORIAL

Malheureusement, je terminerai cet éditorial par un hommage à notre ancien ami Georges Monchaux, membre du Comité de rédaction de notre journal, qui n'a hélas pu bénéficier des progrès et succès de la radiothérapie. Nous garderons au sein du Comité, le souvenir d'un collègue nanti d'une personnalité remarquable d'enthousiasme et de droiture, celui surtout d'un ami.

Henri Métivier

Président du Comité de rédaction 